\title{
A Feasibility Study on the Implementation of Visibility Algorithms for Fault Diagnosis in Aircraft Fuel Systems
}

\author{
Manuel Esperon-Miguez ${ }^{1}$, Adrian Uriondo ${ }^{2}$, Jorge Rodriguez ${ }^{3}$, Bartolo Luque ${ }^{4}$, Ian K. Jennions ${ }^{5}$ \\ 1,2,5 IVHM Centre, Cranfield University, Cranfield, MK43 OAL, United Kingdom \\ ${ }^{1}$ m.esperonmiguez@cranfield.ac.uk \\ 2 a.uriondo@cranfield.ac.uk \\ 5i.jennions@cranfield.ac.uk \\ ${ }^{3,4}$ ETSI Aeronautica y del Espacio, Universidad Politecnica de Madrid, Spain \\ 3jorge.rodriguez@upm.es \\ ${ }^{4}$ bartolome.luque@upm.es
}

\begin{abstract}
This paper discusses the applicability of Visibility Algorithms to detect faults in condition monitoring applications. The general purpose of Visibility Algorithms is to transform time series into graphs and study them through the characterisation of their associated network. Degradation of a component results in changes to the network. This technique has been applied using a test rig of an aircraft fuel system to show that there is a correlation between the values of key metrics of visibility graphs and the severity of four failure modes. We compare the results of using Horizontal Visibility algorithms against Natural Visibility algorithms. The results also show how the Kullback-Leibler divergence and statistical entropy can be used to produce condition indicators. Experimental results show that there is little dispersion in the values of condition indicators, leading to a low probability of false positives and false negatives.
\end{abstract}

\section{INTRODUCTION}

Integrated Vehicle Health Management (IVHM) is aimed at reducing the impact of maintenance activities on operational cost and availability by detecting - and in some cases predicting - faults (Jennions, 2011). This is achieved by measuring physical parameters from a given system using sensors (e.g. pressure in different points of a hydraulic system, vibration of rotating equipment). These data are later analysed using mathematical algorithms to detect and isolate faults (diagnostics) or to calculate the Remaining Useful Life (RUL) of a component (prognostics). These algorithms can be developed using models of the physical phenomena that govern the failure mode (physics-driven methods) or using

Manuel Esperon-Miguez et al. This is an open-access article distributed under the terms of the Creative Commons Attribution 3.0 United States License, which permits unrestricted use, distribution, and reproduction in any medium, provided the original author and source are credited. data analysis techniques to infer detection and isolation rules from large datasets (data-driven methods).

The first generation of automated diagnostic tools was based on an engineering understanding of how each fault manifests different symptoms, and a set of thresholds and logical rules to detect and isolate the fault. Physics-driven methods have evolved from these principles. More recently, data-driven methods have also proven successful at developing diagnostic algorithms.

Hybrid methods combine physics-based and data-driven techniques. A common application of hybrid methods is to use a physics-based model to generate datasets of healthy and faulty conditions when faulty data is scarce or missing. An example this approach is the prognostic tool for multiple wind turbine faults developed to calculate the RUL using a kinematic approach based on the Euclidean distance between clusters of faulty conditions and clusters of normal operation (Djeziri, et al., 2018).

Hybrid methods can also be used to provide a diagnosis with incomplete information, like the hybrid hierarchical diagnosis method developed by Yawei, Mingqing, Zhao, Lei and Yajun (2018) or the technique based on the principle of analytical redundancy that Benmoussa and Djeziri (2017) demonstrated on a mechanical transmission.

Data-driven methods used in diagnostics can be divided into the following categories (Stutz, 2010):

- Classification techniques, which are used to generate a classification model from training data that can distinguish between healthy and faulty states as well as isolate faults. Some of the techniques included in this group are Neural Networks (NN), Gaussian processes, Bayesian classification, support vector machines, and rule-based classification. 
- Nearest neighbour detection techniques, which work under the assumption that datasets form dense neighbourhoods of data points and that anomalies result in deviations from them, which can be analysed based on the distance to other neighbours and the density of the data points.

- Clustering, which are unsupervised techniques aimed at classifying data into natural classes. Examples of clustering include, among many others, Self-Organizing Maps (SOM), k-means clustering, and probabilistic clustering via Expectation Maximization (EM) optimization

- Classical statistical techniques, which work by approximating different phenomena to probability distributions whose parameters are calculated based on samples from the dataset.

Another classification for data-driven methods is based on the nature of the dataset (Gao, et al., 2015): time-domain, frequency-domain, and time-frequency-domain.

A new set of methods used to analyse time-domain signals and extract information from their changes are Visibility Algorithms (Lacasa, et al., 2008). Visibility Algorithms transform, according to a geometric criterion, time series into networks (named Visibility Graphs) which can later be analysed using graph theory to extract valuable information from the original time series. This technique has proven effective, for example, to detect periodicity (Núñez, et al., 2011), measure fractality (Lacasa, et al., 2009), analyse irreversibility (Lacasa, et al., 2012) or distinguish between chaotic and stochastic time series (Lacasa \& Toral, 2010). Beyond theoretical matters, Visibility Algorithms have recently been used to analyse seismicity (Aguilar-San Juan \& Guzman-Vargas , 2013), frequency of hurricanes (Elsner, et al., 2009), diagnose Alzheimer's disease (Ahmadlou \& Adeli, 2010) or to characterise the texture of milled surfaces (Sanz-Lobera, et al., 2015).

With their ability to analyse signals without focusing on changes suffered to their nominal value, Visibility Algorithms present a big potential to distinguish between healthy and faulty states. This paper presents the first application of Visibility Algorithms to develop diagnostic rules and the results. This technique has been applied to detect four typical faults of an aircraft fuel system: filter clogging, faulty pump, pipe clogging, and leaks.

\section{VISIBILITY ALGORITHMS}

\subsection{Horizontal Visibility Algorithm}

The Horizontal Visibility algorithm (HVa) (Luque, et al., 2009; Gutin, et al., 2011) assigns each datum of the series to a node in the graph. Two nodes are connected if a horizontal line can be drawn joining both points without intersections with any intermediate datum (see Figure 1). In other words, let $\left\{x_{i}\right\} i=1, \ldots, N$ be a time series of $\mathrm{N}$ real data. Two nodes $i$ and $j$ in the graph are connected if the following geometrical criterion is fulfilled within the times series $x_{i}$ to $x_{j}$.

$$
x_{i}, x_{j}>x_{n}, \forall n \mid i<n<j
$$

Figure 1 shows how the HVg can be applied to a time signal, where each data point is represented as a bar. Each datum in the series corresponds to a node in the graph (round blue points in the associated circular Visibility Graph) and the red lines represent links between data points (blue links in the Visibility Graph).

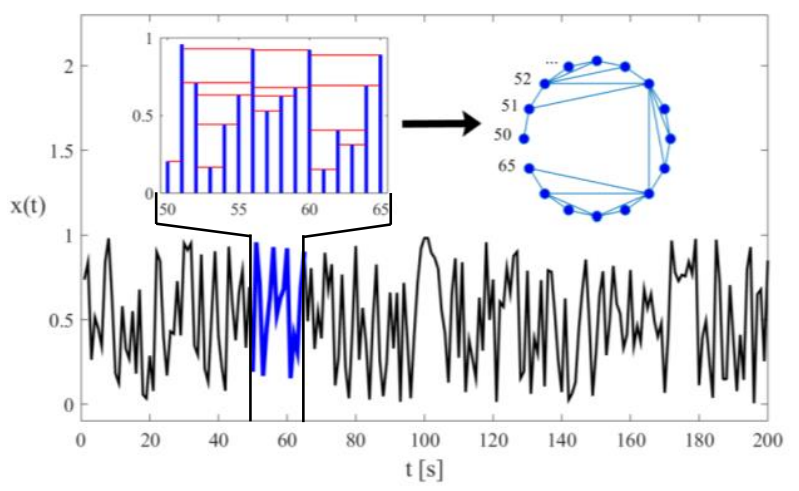

Figure 1. Illustration of the HVa applied on a time signal signal $\mathrm{x}(\mathrm{t})$ from $\mathrm{t}=55 \mathrm{~s}$ to $\mathrm{t}=60 \mathrm{~s}$.
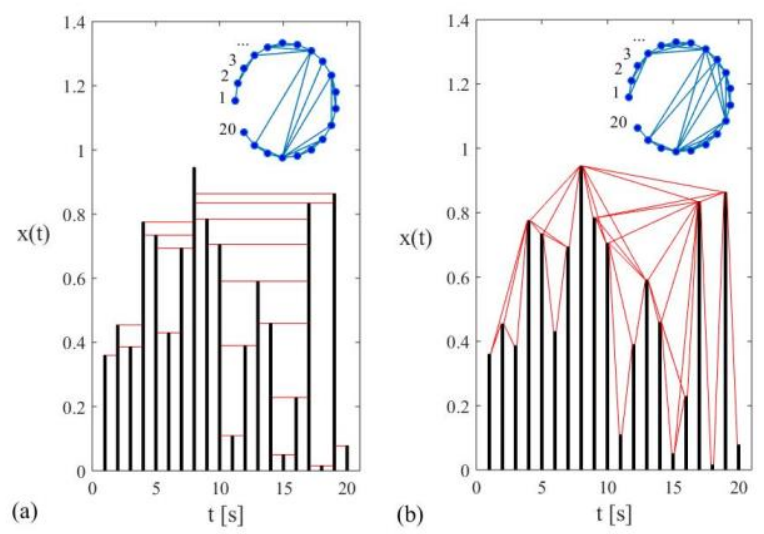

Figure 2. Graphic illustration of the HVa (a), and Natural $\mathrm{NVa}$ (b) applied to the same time series following the geometrical criterion (1) and (2) respectively.

\subsection{Natural Visibility Algorithm}

Like HVa, Natural Visibility algorithm (NVa) (Lacasa, et al., 2008; Lacasa, et al., 2009) assigns each datum of the series to a node in the graph. In this case, two nodes $i$ and $j$ in the graph are connected if a straight line joining $x_{i}$ and $x_{j}$ can be drawn without intersections with any intermediate data point (see Figure $2 \mathrm{~b}$ ). In other words, $i$ and $j$ are two connected if the following geometrical criterion is fulfilled within the time series: 


$$
x_{n}<x_{i}+\frac{n-i}{j-n}\left(x_{j}-x_{i}\right) \quad \forall n \mid i<n<j
$$

The HVg derived from a given signal will have lower number of links than the associated $\mathrm{NVg}$ because its visibility criterion is more restrictive. In fact, the $\mathrm{HVg}$ will be a subgraph of the $\mathrm{NVg}$, meaning that that $\mathrm{NVgs}$ are more sensible to long correlations. In contrast, the $\mathrm{HVg}$ is less expensive computationally and more tractable analytically (Lacasa, et al., 2009).

Lacasa et al. (2008) have shown that time series structures are inherited in the associated graph, such that periodic, random, and fractal series map into motif-like, random exponential and scale-free networks respectively (Newmann, 2003) (Newman, et al., 2006). These findings indicate that the graph might capture the dynamic fingerprints of the process that generated the series. This suggests that Visibility algorithms might be able to extract additional information from signals regarding the operational conditions of the system. Consequently, these algorithms were put to the test and used to develop diagnostic rules for an aircraft fuel system.

\section{USE CASE: AIRCRAFT FUEL SySTEM}

The main function of an aircraft fuel system is to supply a given flow of fuel to the engines at a certain pressure. For aircraft of a certain size, the fuel system must also be able to transfer fuel between different tanks to ensure the aircraft's centre of gravity is within specified limits. In some cases, the fuel can be used to dissipate heat from the oil used for lubrication.

Detecting faults in a fuel system is essential to guarantee the safe operation of the aircraft. Isolating the component responsible for the fault is equally important since the aircrew must make critical decisions to respond in an appropriate manner. This information is also crucial for maintainers because troubleshooting can become a significant portion of the time dedicated to maintenance tasks.

This use case focuses on four critical failures in fuel systems:

- $\quad$ Filter clogging

- $\quad$ Faulty pump

- Leaks

- $\quad$ Pipe clogging

Whilst fuel systems can suffer other faults besides those listed here, this is a compilation of some of the most common. This approach is consistent with the work published by (Niculita, et al., 2012; Niculita, et al., 2013; Niculita , et al., 2014), who used the same test rig to develop and test different IVHM solutions for aircraft fuel systems.

This analysis is not focused on a specific aircraft model and, consequently, it considers the effects of these failures on a generic fuel system. Such system must include: pumps, valves, filters, tanks, pipes, pressure sensors, and flow sensors.

\subsection{Test Rig}

Data for this study were collected using a scaled fuel test rig which proportional control valves to simulate the effects of each fault listed above (Figure 3). The rig measures the pressure on different locations as well as the volumetric flow through the exit nozzle. The rig uses water instead of fuel because it is safer to operate, although this does not affect its ability to be used as a demonstrator of how faults result in deviations of pressure and mass flows in the system.

Filter clogging and pipe clogging induce a pressure drop in the system and therefore are replicated in the rig using proportional valves to increase the resistance to the flow of water. In the case of pumps, the main failures they can suffer are recirculation and leaking, both of which can be simulated by allowing part of the fluid to leave the main branch of the circuit and return to the tank. The same can be said for replicating a leak, which is achieved by allowing part of the flow to escape by opening a valve thus reducing the flow through the main circuit.

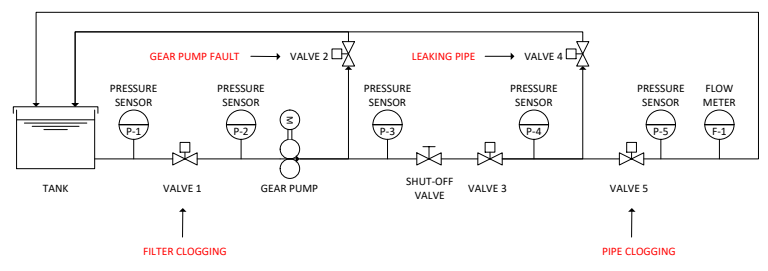

Figure 3. Diagram of the fuel system test rig. Failure modes (in red) are simulated using the valve indicated in each case.

\subsection{Description of tests}

The aim of the first set of tests was to collect data on steady state conditions for different faults with different degrees of severity. Each failure mode was tested independently, meaning that one valve was used to simulate the failure and the rest remained in their default position - consistent with a fault free system - during each test. Failures were simulated with increasing levels of severity by changing the position of the valve in intervals of $10 \%$, with $100 \%$ being completely open, and $0 \%$ complete closed (Table 1). In total, this first set of tests include 41 different cases.

\begin{tabular}{|c|c|c|c|c|}
\hline \multirow[b]{2}{*}{ Failure Mode } & \multirow[b]{2}{*}{ Valve } & \multicolumn{2}{|c|}{ Range of valve positions } & \multirow[b]{2}{*}{$\begin{array}{l}\text { No. } \\
\text { cases }\end{array}$} \\
\hline & & Totally Healthy & $\begin{array}{c}\text { Maximum } \\
\text { Failure Severity }\end{array}$ & \\
\hline Filter clogging & 1 & $100 \%$ & $0 \%$ & 11 \\
\hline Pump failure & 2 & $0 \%$ & $100 \%$ & 11 \\
\hline Leaks & 4 & $0 \%$ & $100 \%$ & 11 \\
\hline Pipe clogging & 5 & $100 \%$ & $10 \%$ & 10 \\
\hline
\end{tabular}

Table 1. List of failure modes for first set of tests. The position of each valve was modified by $10 \%$ on each test 
Completely closing valve 5 , with the rest remaining in their default position, would result in the pressure reaching values that could damage some of components of the rig. Consequently, tests for pipe clogging were stopped before reaching total failure conditions.

The parameters measured during each test include the pressure of five different points of the circuit and the volumetric flow at the exit nozzle. Whilst the rotational speed of the pump was also captured, it remained at 400rpm in all of them.

The sampling frequency of all sensors was $1 \mathrm{kHz}$. Signals were not filtered, or subjected to any kind of post-processing. Visibility algorithms tend to require datasets that have 10,000 data points or more to be able to differentiate between different operating conditions (Lacasa, et al., 2009) (Lacasa $\&$ Toral, 2010). Consequently, each dataset corresponds to approximately 10 seconds of testing. This proved sufficient to discriminate between healthy and faulty conditions. Had this not been the case, the sample size would have had to be increased.

\begin{tabular}{lcrr}
\multirow{2}{*}{ Failure Mode } & \multirow{2}{*}{ Valve } & \multicolumn{2}{c}{ Valve position } \\
\cline { 3 - 4 } & & Healthy & \multicolumn{1}{c}{ Faulty } \\
\hline Filter clogging & 1 & $80 \%$ & $30 \%$ \\
Faulty pump & 2 & $30 \%$ & $70 \%$ \\
Leaks & 4 & $0 \%$ & $50 \%$ \\
Pipe clogging & 5 & $80 \%$ & $20 \%$
\end{tabular}

Table 2. Valve positions for health and faulty conditions for second set of tests

A second set of tests were conducted to get sufficient data points to analyse the probability distribution of results obtained for healthy and faulty conditions. These data are necessary to determine the accuracy of a diagnostic tool, i.e. the probability of getting a false positive or false negative.

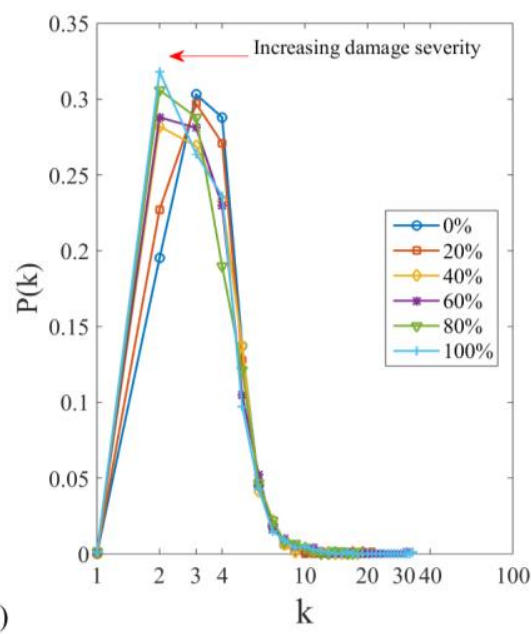

Table 2 shows the valve positions corresponding to health and faulty conditions of each failure mode $(8$ different conditions in total). Each of these conditions was tested 31 times (248 tests in total) and each test collected 10,000 data points at $1 \mathrm{kHz}$. Developing diagnostic rules

Each sensor produces a signal that is stored as a vector of data which is then transformed into a visibility graph using $\mathrm{HVa}$ or NVa. The visibility graph of a signal suffers changes if the operating conditions of the system are altered, i.e. the position of a valve changes. If a change to the visibility graph can be unequivocally linked to a failure mode, it is possible to develop a diagnostic rule.

A technique that has proven useful in the past is to study the changes suffered by the probability distribution of the connectivity of the graph, $P(k)$ (Lacasa, et al., 2008) (Newmann, 2003) (Newman, et al., 2006). This curve indicates what proportion of data has visibility $\mathrm{k}$ of the other data. Figure 4 shows how $P(k)$ of the HVg and NVg from the time series measured by sensor $\mathrm{P}_{4}$ change shape as the severity of a pump fault increases.

The next step is to define a Condition Indicator (CI) based on the changes experienced by these probability distributions. In this paper we will discuss two different approaches. The first CI uses the Kullback-Liebler divergence (Kullback \& Leibler, 1951), $\mathrm{D}_{\mathrm{KL}}$, to measure the difference between the probability distribution of connectivity for healthy conditions, $P H(k)$, and that obtained with a different valve position, $P(k)$ :

$$
D_{K L}\left(P P_{H}\right)=\sum_{k} P(k) \ln \frac{P(k)}{P_{H}(k)}
$$

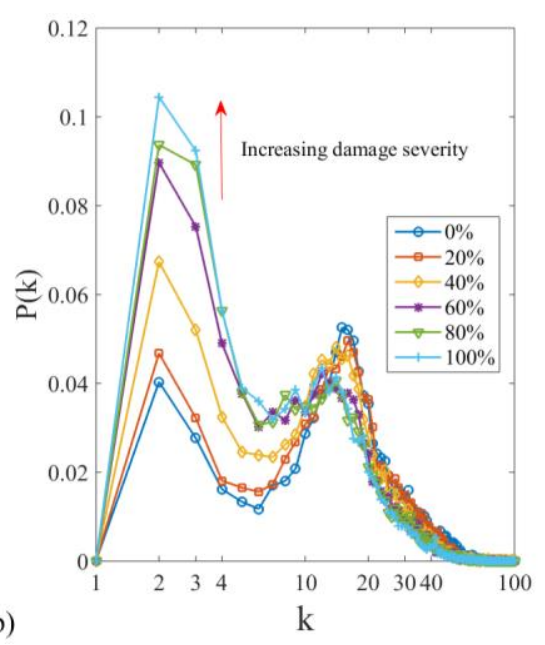

Figure 4. Faulty pump: evolution of the probability distribution of the connectivity of the HVg (a) and NVg (b) generated using the signal from sensor $\mathrm{P}_{4}$. Each curve corresponds to a test with valve 2 at a different positions with $20 \%$ intervals. 
(a)

(b)
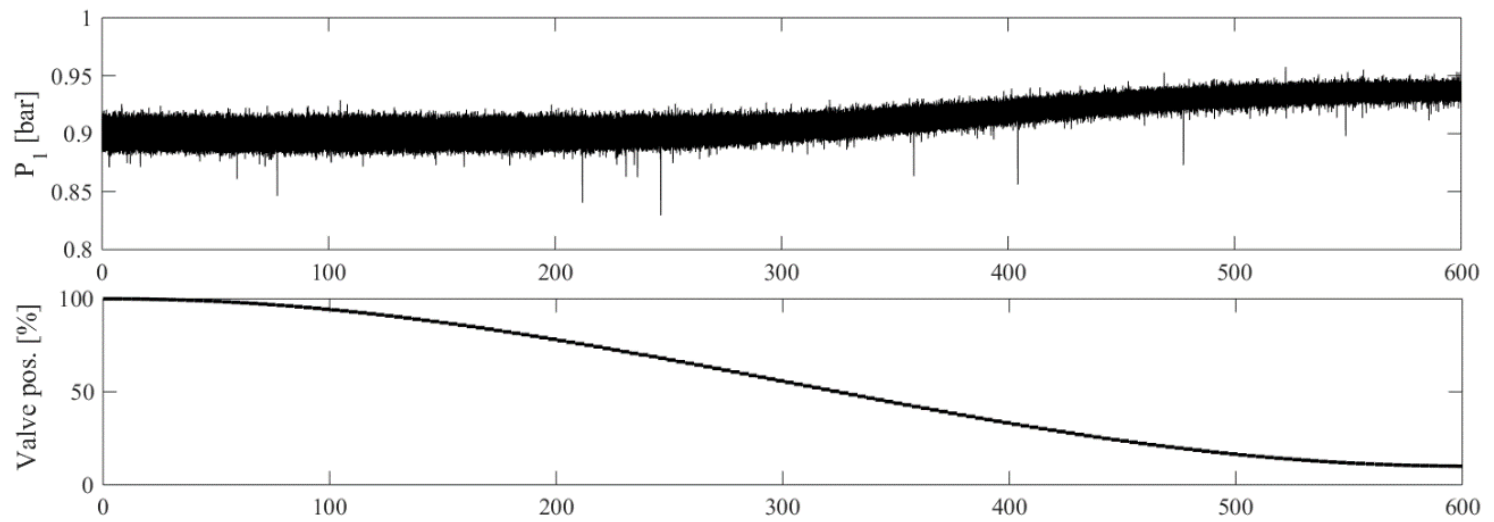

(c)

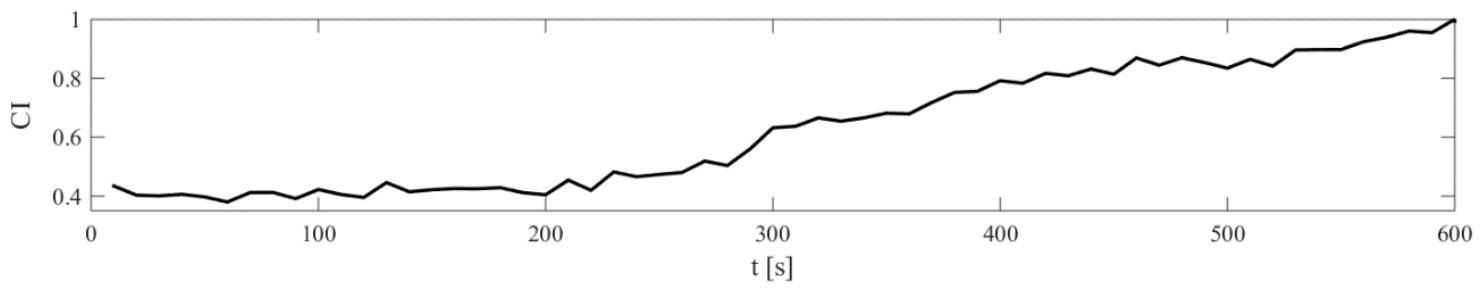

Figure 5. Evolution of the signal of sensor $\mathrm{P}_{1}$ (a) as valve 1 is closed to simulate the clogging of a filter (b), and the evolution of a dimensionless $\mathrm{CI}$ using the KL divergence applied to the $\mathrm{HVg}$ of the signal (c).

Using $D_{K L}$ means that the CI is referenced against a healthy estate and its value can be interpreted as a measurement of the deviation between the system's current state and its healthy state.

The second CI used in this study uses the entropy, $H$, to study changes in $P(k)[14,15]$ :

$$
H=-\sum_{k} P(k) \ln P(k)
$$

Whilst $H$ can be calculated for a probability distribution in isolation, a CI works better if it uses a reference to compare the current estate of the system to a healthy estate. Consequently, the second CI uses the entropy of a healthy state, $H_{H}$, as reference and is calculated using the following formula:

$$
\widehat{\Delta H}=\frac{\Delta H}{H_{H}}=\frac{H-H_{H}}{H_{H}}=\frac{\sum_{k}\left(P(k) \ln P(k)-P_{H}(k) \ln P_{H}(k)\right)}{\sum_{k} P_{H}(k) \ln P_{H}(k)}
$$

These CI do not rely on changes in the amplitude of the signals. Instead, they highlight changes in the shape of the signal by using Visibility Algorithms.

Figure 5a shows how the value of $\mathrm{P}_{1}$ changes as the filter clogging worsens, which is simulated closing valve 1 as shown in Figure 5b. Apparently, the pressure signal contains no information about the clogging state, as it changes less than $5 \%$ for the whole range of the valve positions. The graph at the bottom (Figure 5c) shows the result of applying a $\mathrm{NVa}$ to $\mathrm{P}_{1}$ and using the normalised value of $D_{K L}$ as $\mathrm{CI}$. The value of $\mathrm{CI}(t)$ was calculated with 10,000 values of $\mathrm{P}_{1}$ using a time window from $t$ - $10 \mathrm{~s}$ to $t$ (hence the $10 \mathrm{~s}$ gap at the beginning of the curve). This example illustrates how a CI based on Visibility Algorithms can be correlated with the severity of a fault and its value change more significantly than the signal on which it is based.

\section{RESULTS AND DISCUSSION}

\subsection{Changes in the NVg of each signal for each failure mode}

Data from the first set of tests were transformed using NVa and changes in their $P(k)$ analysed using the Kullback-Liebler divergence (Figure 6.) Experiments show that filter clogging and pipe clogging see the biggest change in absolute value of $D_{K L}$ thanks to the sudden increase of $\mathrm{P}_{4}$ and $\mathrm{P}_{5}$ with high fault severity. However, it must be noted that these sudden increases are the result of extreme system degradation: completely blocking the inlet of water to the system (by closing vale 1 completely) or almost completely closing the outlet (with valve 5 at $10 \%$ ) whilst the pump continues to force water into the system. This produces shockwaves that travel through the system, distorting the signals, and leading to higher values of $D_{K L}$.

It is important to note that there are several examples of nonlinear behaviour for high levels of severity. For filter clogging the $D_{K L}$ of $\mathrm{P}_{4}$ and $\mathrm{P}_{5}$ drops starts dropping once the valve position drops below $30 \%$ and therefore are not a good basis for a CI. Similarly, pipe clogging sees a $35 \%$ drop in $D_{K L}$ of $\mathrm{P}_{3}$. Less pronounced is the non-linear behaviour of $\mathrm{P}_{3}$ and $\mathrm{Q}$ for pump faults and leaks in the system. Since these non-linearities appear as the severity of the fault increases it is possible that shockwaves in the system are the cause. However, other sources of signal noise cannot be discarded. 
In the case of filter clogging we see that $\mathrm{P}_{1}, \mathrm{P}_{3}$, and $\mathrm{Q}$ present a higher variation with the increasing severity of the fault. The change in value of $D_{K L}$ for these three signals is still significant enough to differentiate between heathy and faulty conditions. This is quite remarkable, because the sensor reading $\mathrm{P}_{1}$ is located upstream of the valve and yet the $D_{K L}$ of its associated $\mathrm{NVg}$ provides a better indication of degradation than $\mathrm{P}_{2}$, which is located downstream.

Pump fault results are well correlated with the $D_{K L}$ of $\mathrm{P}_{3}, \mathrm{P}_{4}$, $\mathrm{Q}$, and $\mathrm{P}_{5}$, although the variation of the latter is smaller than for the first four. All of these parameters see a sudden increase with the valve open at $30 \%$ or $40 \%$ and reach 50 $60 \%$ of their maximum value with a valve position of $50 \%$. This means that this is a good indicator of early degradation.

Filter clogging and pipe clogging are simulated by restricting the flow through the fuel rig at different points, hence the similarity between Figure $6 \mathrm{a}$ and Figure 6c. However, changes in the $D_{K L}$ of the NVg of each signal are noticeable for a narrower range of valve positions: between $30 \%$ and $10 \%$ (the latter being a limit imposed by the configuration of the rig). Conversely, filter clogging shows clear indications of degradation since $40 \%$ until the valve is totally closed.

Leaks reduce the mass flow and the dynamic pressure in the main branch of the fuel rig. This is a similar mechanism to the way pump faults are simulated, and yet $\mathrm{P}_{3}$ and $\mathrm{Q}$ show
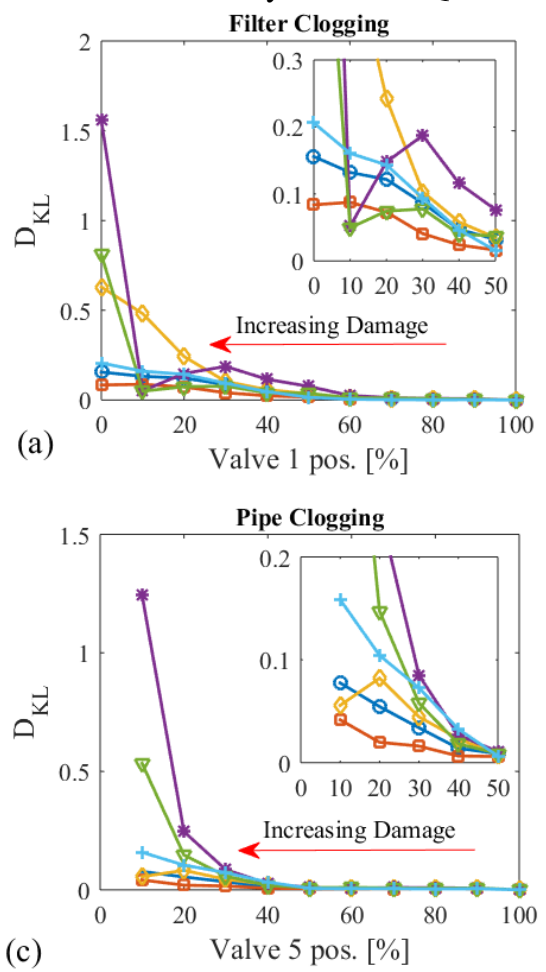

(b)

much greater changes than sensors located downstream from the leak (i.e. $\mathrm{P}_{4}$ and $\mathrm{P}_{5}$ ). This reinforces the hypothesis that dynamic effects such as shockwaves are being picked up by the visibility algorithm.

These results indicate that for each failure mode there are signals whose $\mathrm{NVg}$ is highly correlated with the condition of the component and that Visibility Algorithms can be used as the basis of diagnostic algorithms. The next step is to identify which signals will be used as inputs to detect and isolate each fault. Comparing Visibility Algorithms and condition indicators

\subsection{Comparing Visibility Algorithms and condition indicators}

If a diagnostic system is to be used to monitor the condition of several components simultaneously, it is not possible to use the same CI for more than one type of fault. Otherwise, the system could not differentiate between two different failures, leading to false positives. Furthermore, to unequivocally link a CI to a particular failure mode, the CI cannot suffer a similar change for more than one failure mode. An example of this problem can be seen in the $D_{K L}$ of the NVg of Q, which is a clear indicator of a deviation from healthy conditions for every single failure modes (Figure 6) making it impossible to isolate a fault using this CI.
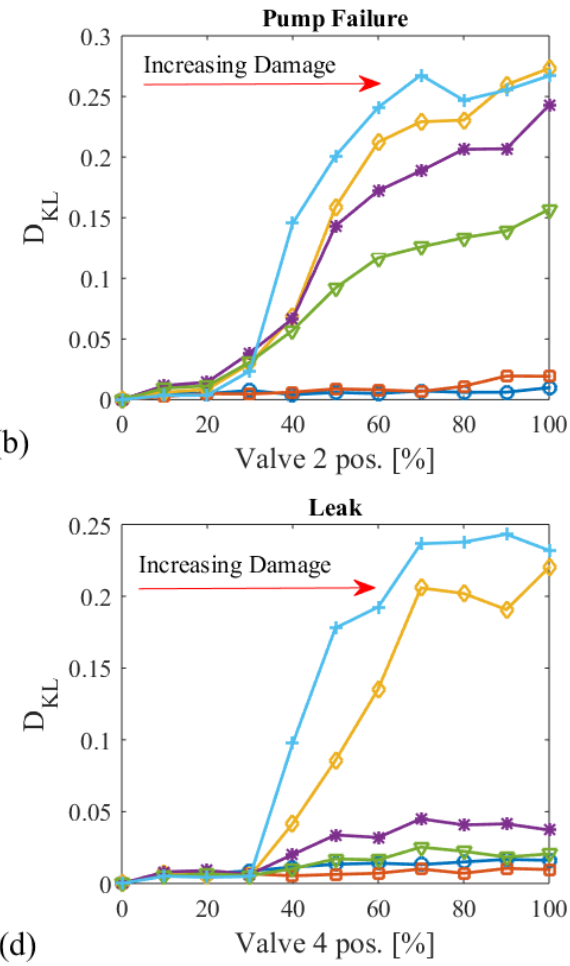

(d)

$$
\multimap \mathrm{P}_{1} \longrightarrow \mathrm{P}_{2} \multimap \mathrm{P}_{3} \rightarrow \mathrm{P}_{4} \rightarrow \mathrm{P}_{5} \longrightarrow \mathrm{Q}
$$

Figure 6. Evolution of the Kullback-Liebler divergence applied to the NVg of each signal for each failure mode: filter clogging (a), faulty pump (b), pipe clogging (c), and leaks (d). 
From a condition monitoring perspective, signals that show a smaller but consistent increase of $D_{K L}$ are more useful to develop diagnostic algorithms because they can be used to detect faults when they are less severe. An example of this phenomenon is how for filter clogging the $D_{K L}$ of the $\mathrm{NVg}$ of $\mathrm{P} 4$ has a spike when the filter is fully clogged (i.e. the valve is totally closed), but has a lower value than P1, P3, P5, and $\mathrm{Q}$ for less severe fault conditions.

From a systems engineering perspective, using as few signals as possible means fewer sensors, less wiring, lower weight and cost, and a much simpler monitoring system. For this reason, even though it is not uncommon to use combinations of signals as a CI (e.g. the difference between inlet and outlet pressure of a filter), we decided to focus on CI that use one signal as input.

Figure 7 shows the values of $D_{K L}$ and $\widehat{\Delta H}$ of both the $\mathrm{HVg}$ and $\mathrm{NVg}$ of signals chosen to detect each fault: P1 for filter clogging, P4 for pump failure, and P3 for pipe clogging and leaks. Whilst both pipe clogging and leaks use P3, it is still possible to differentiate between both failure modes because the entropy takes negative values for the former and positive values for the latter. The choice of signals as inputs for CI was made based on those listed above.

These results show that both $D_{K L}$ and $\widehat{\Delta H}$ are clearly correlated with all failure modes. However, there is no clear advantage of one over the other that can be generalised to other applications. Conversely, when looking at the results obtained using different Visibility Algorithms we see that
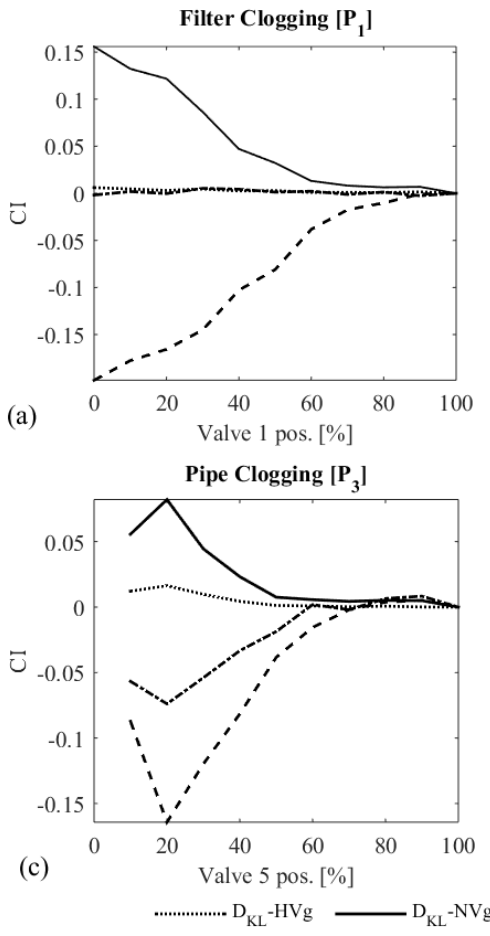

$\mathrm{NVa}$ result in a greater differentiation between healthy and faulty conditions than HVa, although CIs calculated using $\mathrm{HVa}$ are still well correlated with each fault. This is the result of NVa producing graphs with more information about a given signal than $\mathrm{HVa}$, although $\mathrm{HVa}$ requires significantly less computer power and might present advantages when processing power is at a premium.

Looking at the values of $\mathrm{P}_{3}$ for Pipe Clogging and Leak in Figure 7 it is noticeable that the non-linear behaviour that was first noticed in Figure 6 appears here as well. This is not surprising for the $D_{K L}$ of the NVg because Figure 7 shows the normalised values of the data presented earlier. However, these graphs indicate that the transition in slope happens at the same level of degradation with data processed using $\mathrm{NVa}$ and $\mathrm{HVa}$ and post-processed with either $D_{K L}$ or $\widehat{\Delta \mathrm{H}}$ (the normalisation makes it less noticeable for Leaks processed with $\mathrm{HVa}$ but it still happens.) The explanation for this change in slope is that for $\mathrm{P}_{3}$ in Pipe Clogging and Leak the evolution of the probability distribution of the connectivity as the severity increases changes direction (see Figure 4).

What these graphs also show is that non-linearities are independent of which Visibility Algorithm is used or how the visibility graph is post-processed. The fact that the changes in normalised values are higher for $\mathrm{NVa}$ is consistent with $\mathrm{NVa}$ resulting in a higher number of nodes with higher connectivity compared to applying a HVa to the same time series.
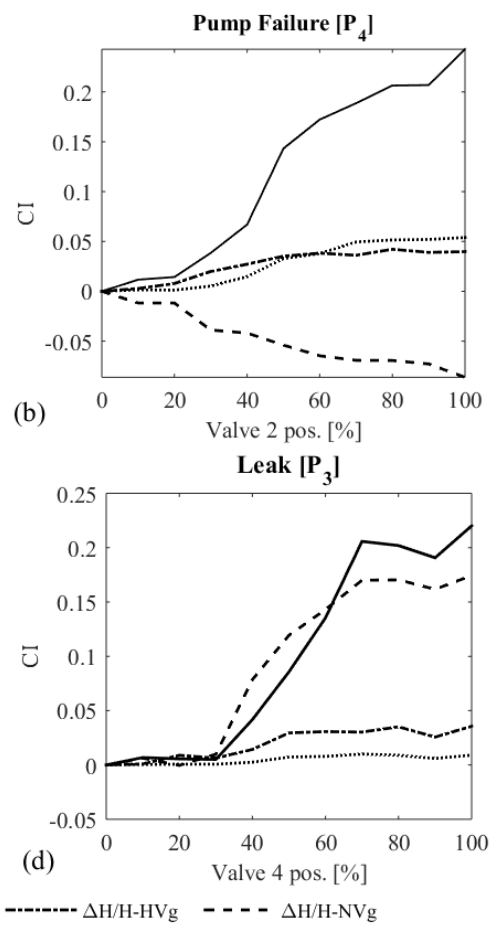

Figure 7. Normalised values of $D_{K L}$ and $\widehat{\Delta H}$ using $\mathrm{HVa}$ and $\mathrm{NVa}$ for the value of $\mathrm{P}_{1}$ in filter clogging (a), $\mathrm{P}_{4}$ in pump failure (b), $\mathrm{P}_{3}$ in pipe clogging (c), and $\mathrm{P}_{3}$ in leaks (d). 


\subsection{Analysing the accuracy of diagnostic rules}

A diagnostic algorithm must be able to differentiate between a system that is operating as planned and deviations that indicate that a component has failed (the valve positions corresponding to each of these conditions are included in Table 2. This is done by setting a threshold for the value of the CI that, if reached, triggers an alarm indicating that the component has failed. So far we have proved that the values of CI based on Visibility Algorithms do change as the fault becomes more severe.

Numerous factors contribute to fluctuations in the readings of any diagnostic system: sensor noise, changes to operating conditions, vibrations, hysteresis in actuators, numerical approximations, etc. These are the causes behind false positives and false negatives in IVHM systems. As a result, it is more accurate to assume that the value of any CI in either healthy or faulty conditions is better described as a probability distribution.

The statistical analysis of the results generated with the second set of tests were used to compare the dispersion in the value of CIs calculated using both $D_{K L}$ and $\widehat{\Delta H}$ for $\mathrm{HVa}$ and NVa (Figure 8.) This analysis has shown that CIs calculated using NVg have similar or lower standard deviation than the same CIs calculated using $\mathrm{HVg}$.
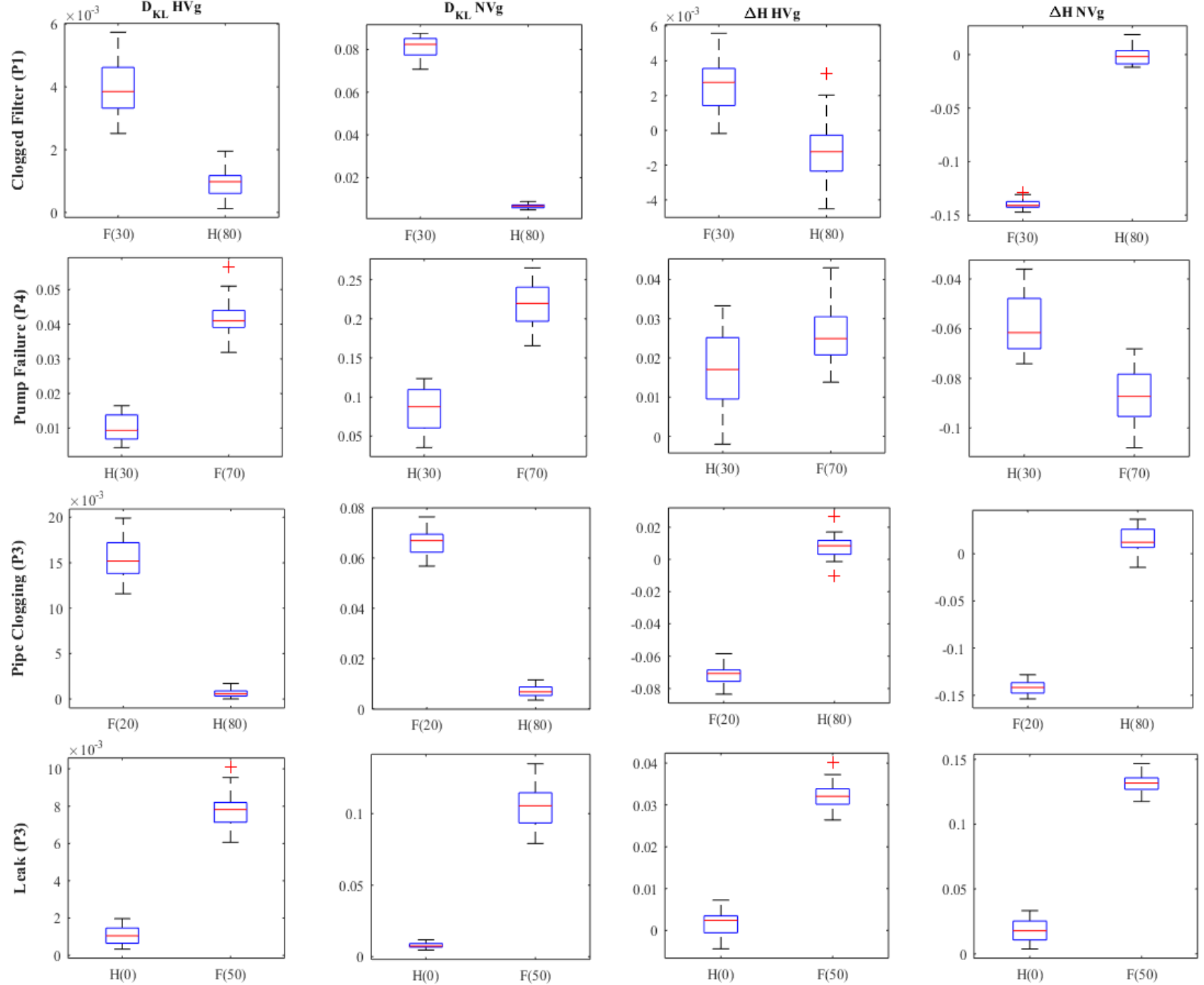

Figure 8. Box plots of the CI calculated using $D_{K L}$ and $\widehat{\Delta H}$ on the $\mathrm{HVg}$ and $\mathrm{NVg}$ for faulty $(\mathrm{F})$ and healthy $(\mathrm{H})$ conditions. On each box, the red central mark is the median, the edges of the blue box are the 25th and 75th percentiles, the whiskers extend

to $+/-2.7 \sigma$, and outliers are plotted individually The valve positions corresponding to healthy and faulty states are in parenthesis. 
INTERNATIONAL JOURNAL OF PROGNOSTICS AND HEALTH MANAGEMENT

\begin{tabular}{|c|c|c|c|c|c|c|c|c|c|}
\hline Failure Mode & Sensor & CI & Condition & $\mu$ & $\sigma$ & Dist. & Threshold & $\mathbf{P}_{\mathrm{FP}}$ & $\mathbf{P}_{\mathrm{FN}}$ \\
\hline \multirow[t]{2}{*}{ Clogged Filter } & \multirow[t]{2}{*}{$\mathrm{P}_{1}$} & \multirow[t]{2}{*}{$\mathrm{D}_{\mathrm{KL}}$ of $\mathrm{NVg}$} & Healthy & 0.0067 & 0.0011 & Weibull & \multirow[b]{2}{*}{0.04} & \multirow{2}{*}{$9 \cdot 10^{-11}$} & \multirow{2}{*}{$5.16 \cdot 10^{-8}$} \\
\hline & & & Faulty & 0.0814 & 0.0044 & Weibull & & & \\
\hline \multirow[t]{2}{*}{ Pump Failure } & \multirow[t]{2}{*}{$\mathrm{P}_{4}$} & \multirow[t]{2}{*}{$\mathrm{D}_{\mathrm{KL}}$ of $\mathrm{NVg}$} & Healthy & 0.0102 & 0.0037 & Weibull & \multirow[b]{2}{*}{0.02} & \multirow[b]{2}{*}{$4.4 \cdot 10^{-3}$} & \multirow[b]{2}{*}{$1.4 \cdot 10^{-3}$} \\
\hline & & & Faulty & 0.0414 & 0.0052 & Weibull & & & \\
\hline \multirow[t]{2}{*}{ Pipe Clogging } & \multirow[t]{2}{*}{$\mathrm{P}_{3}$} & \multirow{2}{*}{$\widehat{\Delta H}$ of $\mathrm{NVg}$} & Healthy & 0.0149 & 0.0124 & Normal & \multirow[b]{2}{*}{-0.05} & \multirow[b]{2}{*}{$8.35 \cdot 10^{-8}$} & \multirow[b]{2}{*}{$9 \cdot 10^{-11}$} \\
\hline & & & Faulty & -0.1419 & 0.0072 & Normal & & & \\
\hline \multirow[t]{2}{*}{ Leaks } & \multirow[t]{2}{*}{$\mathrm{P}_{3}$} & \multirow[t]{2}{*}{$\widehat{\Delta H}$ of $\mathrm{NVg}$} & Healthy & 0.0178 & 0.0084 & Normal & \multirow[b]{2}{*}{0.1} & \multirow[b]{2}{*}{$2 \cdot 10^{-6}$} & \multirow[b]{2}{*}{$9 \cdot 10^{-11}$} \\
\hline & & & Faulty & 0.1313 & 0.0068 & Normal & & & \\
\hline
\end{tabular}

Table 3. Probability distributions of the CI chosen for each failure mode and their corresponding probabilities of false positive and false negative.

These results confirm the entropy of $\mathrm{P}_{3}$ can be used as a CI for both pipe clogging and leaks, showing a clear differentiation between values for healthy and faulty conditions. In the case of filter clogging, $D_{K L}$ shows a small advantage over entropy since it has a smaller standard deviation in healthy conditions, which should result in fewer false positives. Pump failure has the widest spread of results of all the failure modes tested, with a clear overlap in the box plots of healthy and faulty values of CI based on $\widehat{\Delta H}$, hence the choice of $D_{K L}$ as CI for this failure mode as well.

In order to determine the accuracy of a diagnostic tool based on Visibility Algorithms we must calculate the probability of getting false positives, $P_{F P}$, and false negatives, $P_{F N}$. The former is the probability of having a healthy component and a CI whose value has reached the threshold set for the alarm. The latter is the probability having a faulty component whose CI has a value equal or lower than the threshold.

Unlike other data-driven techniques, the methodology shown here does not include an automated way to determine the threshold for the alarms. However, there is no inherent limitation in Visibility Algorithms to automate this step and there are multiple optimisations techniques available that can be applied to determine the optimal alarm thresholds. For this analysis, thresholds were set manually based on the results from experiments.

The analysis of the statistical results represented in Figure 8 where adjusted to probability distribution functions maximising the log-likelihood and checked using a chisquare test with a confidence level set to $95 \%$. As a result, the $D_{K L}$ of the $\mathrm{NVg}$ for clogged filter and pump failures were approximated to Weibull functions, and the entropy of the $\mathrm{NVg}$ of pipe clogging and leaks to normal distributions (Figure 9.) The parameters that characterise these distributions are included in Table 3.

Using these distributions to calculate $P_{F P}$ and $P_{F N}$ showed that Visibility Algorithms can differentiate between healthy and faulty estates with a confidence level of at least $99.5 \%$. Pump faults present the highest $\mathrm{P}_{\mathrm{FP}}$ and $\mathrm{P}_{\mathrm{FN}}$ as expected from the results shown in Figure 9, but all other failure modes present confidence levels of $99.9998 \%$ or higher.

These values are likely to be lower under real flight conditions. However, whilst it must be noted that this analysis does not consider the effect of changing environmental and operating conditions that an aircraft fuel system experiences during a flight, these results show that CIs based on Visibility Algorithms can deal with other typical sources of uncertainty such as sensors noise, dynamic hydraulic effects, valve hysteresis, and vibrations.
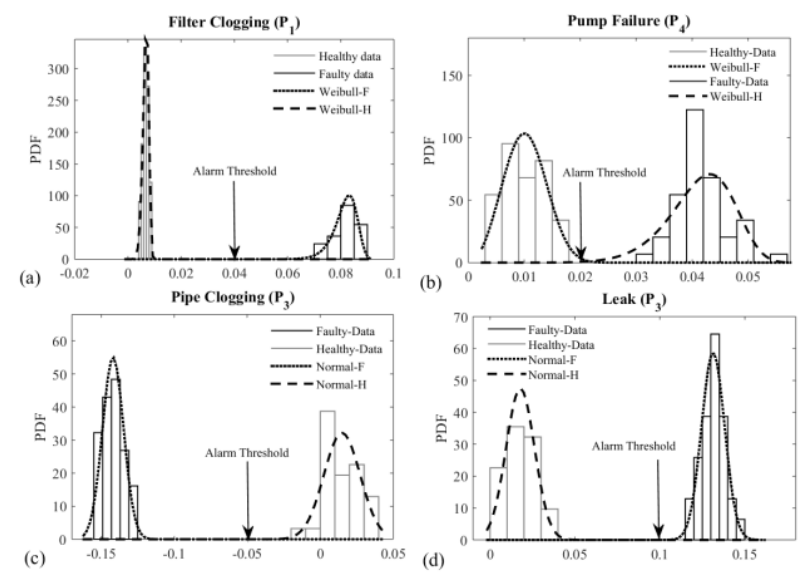

Figure 9. Probability Distribution Functions (PDF) adjusted to the experimental data for healthy and faulty conditions of each failure mode.

\section{Conclusions}

In this work we have applied Visibility Algorithms to study the data from a test rig of an aircraft fuel system, defining a CI based on the Kullback-Leibler divergence and the entropy of the probability distributions that the algorithms generated, and we have conducted a statistical study to validate the CI selected

The results presented in this paper show that changes to the visibility graph associated to a sensor reading are correlated to changes in the system and can be used to differentiate 
between faulty and healthy conditions. Since Visibility Algorithms do not require signal processing this can result in fewer steps between data acquisition and diagnosis generation

Experiments show that CIs based on NVg experience greater changes between healthy and faulty conditions than those based on HVg. They also have similar or lower standard deviations, leading to more accurate diagnoses (i.e. lower false positive and false negative rates.) However, it is important to highlight that HVas demand fewer computational resources, which can be advantage in certain applications.

Both the Kullback-Leibler divergence and $\widehat{\Delta H}$ can be used as a CI, without any clear advantage of one over the other. Using different ways to calculate the value of a CI using the same signal as input can lead to using fewer sensors than the number of failure modes that have to be monitored. This is demonstrated by the fact that signals from 3 sensors (P1, P3, and $\mathrm{P} 4$ ) are used to detect 4 failure modes.

The low values of the probabilities of false positives and false negatives indicate that Visibility Algorithms are not particularly susceptible to the inherent uncertainties in the system. Since the current noise level does not seem to affect the ability of Visibility Algorithms to distinguish between faulty and healthy conditions, this method could make signal filtering in diagnostics redundant.

Future works should delve into the definition and utilisation of CIs in order to develop a set of CIs that extract maximum information from a given signal through the use of Visibility Algorithms.

The choice of 10,000 data points was based on previous work published on visibility algorithms and proved to be enough to detect faults with a high level of accuracy. However, further work is needed to understand the sensitivity of diagnosis accuracy to the dataset size for each scenario and how to determine the optimal sample size.

\section{NOMENCLATURE}

CI Condition Indicator

EM Expectation Maximization

HVa Horizontal Visibility Algorithm

HVg Horizontal Visibility graph

IVHM Integrated Vehicle Health Management

NN Neural Networks

$\mathrm{NVa} \quad$ Natural Visibility Algorithm

$N V g \quad$ Natural Visibility graph

RUL Remaining Useful Life

SOM Self-Organizing Maps

\section{REFERENCES}

Aguilar-San Juan , B. \& Guzman-Vargas , L., 2013. Earthquake magnitude time series: scaling behavior of visibility networks. Eur Phys $J$ B, p. 86.

Ahmadlou, M. \& Adeli, H., 2010. New diagnostic EEG markers of the Alzheimer's disease using visibility graph. J Neural Transm, p. 117:1099-109.

Benmoussa, S. \& Djeziri, M. A., 2017. Remaining useful life estimation without needing for prior knowledge of the degradation features. IET Science, Measurement \& Technology, 11(8), p. $1071-1078$.

Djeziri, M. A., Benmoussa, S. \& Sanchez, R., 2018. Hybrid method for remaining useful life prediction in wind turbine systems. Renewable Energy, Volume 116, pp. 173-187.

Elsner, J. B., Jagger, T. H. \& Fogarty, E., 2009. Visibility network of United States hurricanes. Geophys Res Lett, p. 36:L16702.

Gao, Z., Cecati, C. \& Ding, S. X., 2015. A Survey of Fault Diagnosis and Fault-Tolerant Techniques-Part I: Fault Diagnosis With Model-Based and Signal-Based Approaches. IEEE Transactions on Industrial Electronics, 62(6), pp. 3757 - 3767.

Gutin, G., Mansour, T. \& Severini, S., 2011. A characterization of horizontal visibility graphs and combinatorics on words. Phys A Stat Mech Its Appl, p. 390:2421-8.

Jennions, I. K., 2011. Integrated Vehicle Health Management: Perspectives on an Emerging Field. s.l.:SAE International.

Kullback, S. \& Leibler, R., 1951. On Information and Sufficiency. Ann Math Stat, p. 22:79-86.

Lacasa, L., Luque, B., Luque, J. \& Nuño, J. C., 2009. The visibility graph: A new method for estimating the Hurst exponent of fractional Brownian motion. EPL Europhysics Lett, p. 86:30001.

Lacasa, L., Luque, L., Ballesteros, F. \& Nuño, J. C., 2008. From time series to complex networks: the visibility graph. Proc Natl Acad Sci U S A, p. 105:4972-5.

Lacasa, L. et al., 2012. Time series irreversibility: A visibility graph approach. Eur Phys J, p. 85.

Lacasa, L. \& Toral, R., 2010. Description of stochastic and chaotic series using visibility graphs. Phys Rev E - Stat Nonlinear, Soft Matter Phys, p. 82.

Luque, B., Lacasa, L., Ballesteros, F. \& Luque, J., 2009. Horizontal visibility graphs: Exact results for random time series. Phys Rev E - Stat Nonlinear, Soft Matter Phys, p. 80.

Newman, M., Barabasi, A. L. \& Watts, D. J., 2006. The Structure and dynamics of networks. s.l.:Princeton University Press.

Newmann, M. E., 2003. The Structure and Function of Complex Networks. SIAM, p. 45:167-256. 
Niculita , O., Skaf, Z. \& Jennions, I. K., 2014. The Application of Bayesian Change Point Detection in UAV Fuel Systems. s.1., s.n., p. 22:115-21.

Niculita, O., Irving, P. \& Jennions, I. K., 2012. Use of COTS Functional Analysis Software as an IVHM Design Tool for Detection and Isolation of UAV Fuel System Faults. s.l., s.n., pp. vol. 3, p. 13.

Niculita, O., Jennions, I. K. \& Irving, P., 2013. Design for diagnostics and prognostics: A physical-functional approach. s.1., s.n., p. 1-15.

Núñez, A. M. et al., 2011. Detecting series periodicity with horizontal visibility graphs. J Bifurc Chaos, p. 1-8.

Sanz-Lobera, A., Gonzalez, I., Rodriguez, J. \& Luque, B., 2015. Feasibility Study for Visibility Algorithms Implementation in Surface Texture Characterization. Barcelona, s.n.

Stutz, J., 2010. On Data-centric Diagnosis of Aircraft Systems. IEEE Trans Syst Man Cybern - PART C .

Yawei, G. et al., 2018. A hybrid hierarchical fault diagnosis method under the condition of incomplete decision information system. Applied Soft Computing, Volume 73 , pp. $350-365$.

\section{BIOGRAPHIES}

Dr Manuel Esperon-Miguez specialises on design of IVHM systems for new and legacy platforms. He has been involved in projects to develop new design methodologies for IVHM, condition monitoring for thermal and electromechanical systems, the development of new data driven techniques, combining IVHM with Additive Manufacturing, financial analysis of IVHM solutions, and certification of IVHM systems. In the past he also conducted research on Energy Storage Systems. Dr Esperon has a Master's degree in Mechanical Engineering from the Technical University of Madrid, an MSc in Aerospace Engineering from Brunel University, and a PhD in IVHM from Cranfield University.

Mr. Adrián Uriondo Del Pozo specialises on numerical simulation and system dynamics. He has been involved in projects to develop new health monitoring algorithms for aerospace and mechanical applications. Currently, he works as a rotordynamic Specialist Stress Engineer at Safran Electrical and Power. In the past he also conducted research on Wind Turbine Energy Systems. Adrián Uriondo has a Master's degree in Aerospace Engineering from the Technical University of Madrid, and an MSc by Research from Cranfield University.

Mr Jorge Rodríguez received a BEng in Aerospace Engineering from the Universidad Politécnica de Madrid, Spain in 2016. He is currently working in World Aviation as Quality Manager in a PART 145 organization, head of the RPAS department and pilot instructor.

Professor Bartolo Luque received PhD degree in Physics from the Universidad Politécnica de Cataluña, Barcelona, Spain in 1999 . He is currently a professor at the Department of Applied Mathematics in ETSI Aeronautics and Space of the Universidad Politécnica de Madrid, Spain. His current research interests include complex systems, time series and number theory

Professor Ian K. Jennions' career spans some 40 years, working mostly for a variety of gas turbine companies. He has a Mechanical Engineering degree and a PhD in CFD, both from Imperial College, London. He has worked for RollsRoyce (twice), General Electric and Alstom in a number of technical roles, gaining experience in aerodynamics, heat transfer, fluid systems, mechanical design, combustion, services and IVHM. He moved to Cranfield in July 2008 as Professor and Director of the newly formed IVHM Centre. The Centre is funded by a number of industrial companies, including Boeing, BAE Systems, Thales, Meggitt, MOD, DRS, Alstom Transport and Novartis. He has led the development and growth of the Centre, in research and education, since its inception. He is a Director of the PHM Society, vice-chair of the SAE IVHM Steering Group and contributing member of the IVHM HM-1 committee, on the editorial Board for the International Journal of Condition Monitoring, a Chartered Engineer and a Fellow of IMechE, RAeS and ASME. He is the editor of five recent SAE books on IVHM and a coauthor of the book 'No Fault Found - The Search for the Root Cause', published by SAE in September 2015. 